\title{
Correction to: Robust and blind image watermarking via circular embedding and bidimensional empirical mode decomposition
}

\author{
Xiaochao Wang ${ }^{1} \cdot \mathrm{Kun} \mathrm{Hu}^{2}$ · Jianping $\mathrm{Hu}^{3} \cdot$ Ling $\mathrm{Du}^{4} \cdot$ Anthony T. S. $\mathrm{Ho}^{5} \cdot \mathrm{Hong} \mathrm{Qin}^{6}$
}

Published online: 29 July 2020

(c) Springer-Verlag GmbH Germany, part of Springer Nature 2020

\section{Correction to: The Visual Computer https://doi.org/10.1007/s00371-020-01909-2}

The publication of this article unfortunately contained mistakes. The affiliation and the biography of Ling Du were not correct. The corrected affiliation and biography are given below.

Publisher's Note Springer Nature remains neutral with regard to jurisdictional claims in published maps and institutional affiliations.

Ling $\mathrm{Du}$ is a lecturer in School of Computer Science and Technology at Tiangong University. She received the B.E. and M.E. degrees in Computer Science from Liaoning University, Shenyang, China, and the Ph.D. degree with the School of Computer Science and Technology, Tianjin University in 2016, Tianjin, China. Her current research interests include multimedia information security, digital watermarking, and image processing.
The original article can be found online at https://doi.org/10.1007/ s00371-020-01909-2.

\section{Jianping $\mathrm{Hu}$}

neduhjp307@163.com

Xiaochao Wang

wangxiaochao18@163.com

Kun $\mathrm{Hu}$

ucas_hukun@163.com

Ling Du

duling@tiangong.edu.cn

Anthony T. S. Ho

a.ho@surrey.ac.uk

Hong Qin

qin@cs.stonybrook.edu
1 School of Mathematical Sciences, Tiangong University, Tianjin 300387, China

2 University of Chinese Academy of Sciences, Beijing 100049, China

3 School of Sciences, Northeast Electric Power University, Jilin 132012, China

4 School of Computer Science and Technology, Tiangong University, Tianjin 300387, China

5 Department of Computer Science, University of Surrey, Guildford, Surrey GU2 7XH, UK

6 Department of Computer Science, Stony Brook University, Stony Brook, NY 11794-4400, USA 